\title{
Direct observation of resonant subband-Landau-level coupling in a transport experiment
}

\author{
K. Ensslin, C. Pistitsch, and A. Wixforth \\ Sektion Physik der Universität München, Geschwister-Scholl Platz 1, 8000 München 22, Germany \\ M. Sundaram, P. F. Hopkins, and A. C. Gossard \\ Materials Department, University of California, Santa Barbara, California 93106
}

(Received 16 December 1991)

\begin{abstract}
A parabolic quantum well is investigated by low-temperature transport experiments in tilted magnetic fields. The resonant anticrossing of occupied Landau levels belonging to different subbands is manifested directly in the magnetoresistance. This allows a direct evaluation of the single-particle energy spectrum of the system.
\end{abstract}

The energy spectrum of a two-dimensional electron gas (2DEG) in a magnetic field oriented perpendicular to the 2DEG consists of a series of equidistant Landau levels on each electrical subband. If the magnetic field is tilted with respect to the normal of the sample, Landau levels belonging to different subbands repel each other. This resonant subband-Landau-level coupling (RSLC) has been observed in far-infrared (FIR) transmission experiments on $\mathrm{Al}_{x} \mathrm{Ga}_{1-x} \mathrm{As}$ heterostructures ${ }^{1-4}$ and also on parabolic quantum wells (PQW).$^{5-7}$ In FIR optics intersubband transitions are collective modes and affected by depolarization. ${ }^{8.9}$ While depolarization shifts are small $(-10 \%)$ in usual heterojunctions they dominate the infrared modes in PQWs. For the case of RSLC it has been shown theoretically ${ }^{10}$ that the coupling occurs between the plasma frequency (which is the same as the depolarization shifted intersubband frequency in this case) and the respective Landau levels. This remarkable property of a parabolic potential that has been confirmed experimentally ${ }^{6.7}$ prohibits the direct access of the single-particle energy spectrum by means of optical intersubband spectroscopy.

Here we present magnetotransport experiments that directly reveal the anticrossing of Landau levels belonging to different subbands. This is a powerful technique because in transport experiments the observed properties are always measured at the Fermi energy, $"$ in contrast to optical experiments ${ }^{1-7}$ where transitions from occupied states at the Fermi energy to empty states reveal the level anticrossing. In a transport experiment, this means that the Fermi energy has to lie in the gap between the two repellent states to affect the transport properties. For magnetic fields perpendicular to the plane of the sample the levels can cross and consequently can be degenerate at the Fermi energy. This effect has been observed experimentally ${ }^{12}$ and a missing quantum Hall state has been reported. Here we show that tilting the magnetic field leads to a repulsion of the levels and the quantum Hall state is recovered. The position of the corresponding minimum in the magnetoresistance directly reveals the separation of the single-particle energies of the electric subbands. This very particular situation is highly unlikely in any potential other than a parabolic potential. For a quantitative understanding the subband structure of the parabolic well is calculated self-consistently for $B=0$. The effect of the tilted magnetic field is included in the parabolic approximation. ${ }^{13,14}$ The magnetoresistance is then calculated using the formulas given in the literature (e.g., Ref. 11) and compared with the experiment.

The $\mathrm{GaAs}-\mathrm{Al}_{x} \mathrm{Ga}_{1-x} \mathrm{As}$ parabolic quantum well is grown by molecular-beam epitaxy. The width of the PQW is $75 \mathrm{~nm}$, the carrier density $N_{s}=5 \times 10^{11} \mathrm{~cm}^{-2}$, and the mobility $\mu=100000 \mathrm{~cm}^{2} / \mathrm{V} \mathrm{s}$ at $T=4.2 \mathrm{~K}$. The sample structure is described in detail in Ref. 12. The magnetotransport experiments are performed with lowfrequency $(v \cong 30 \mathrm{~Hz})$ lock-in techniques in a superconducting magnet $(0<B<7 \mathrm{~T})$ at $T=2.2 \mathrm{~K}$. The carrier density $N_{s}$ is controlled via a front gate voltage $V_{g}$. The samples are cooled down in the dark. The sample is mounted on a revolving stage that allows angles of $\alpha= \pm 30^{\circ}$ between the surface normal of the sample and the magnetic field $B$. There are two Hall bars on the surface of the sample oriented perpendicular to each other. When the sample normal is tilted away from the orientation of $B$ one Hall bar is oriented along the axis of rotation, the other one perpendicular to it. Within the accuracy of the experiment the measurements for the two directions of current flow reveal identical results in the investigated regime of temperatures $(T>0.5 \mathrm{~K})$ and angles $\left(\alpha<60^{\circ}\right)$. This confirms the isotropy and high quality of our sample. Furthermore, we conclude that the anisotropic band structure that should arise for large tilt angles ${ }^{15}$ is a minor effect in our experiment.

Figure 1 presents a series of magnetoresistance measurement for various tilt angles $\alpha$. The filling factor is defined by $v=h N_{s} / e B_{\perp}$ where $N_{s}$ is the total carrier density of the system. The degeneracy of a Landau level is determined by the component of the magnetic field $B_{\perp}$ $=B \cos \alpha$, that is perpendicular to the electron gas. ${ }^{13,14}$ The subband index is denoted by $i(i=0,1,2, \ldots)$, the Landau-level index by $n(n=0,1,2, \ldots)$. Thus the total filling factor $v$ is still a well-defined quantity even for tilted magnetic fields, while the subband and Landau-level indices $i$ and $n$ and consequently the carrier densities and filling factors of a given subband are not meaningful any more for $\alpha \neq 0$. For the following discussion the spin splitting of the Landau levels is neglected. It is not observed in the present sample for $T=2.2 \mathrm{~K}$ and $B<6 \mathrm{~T}$ due to the 


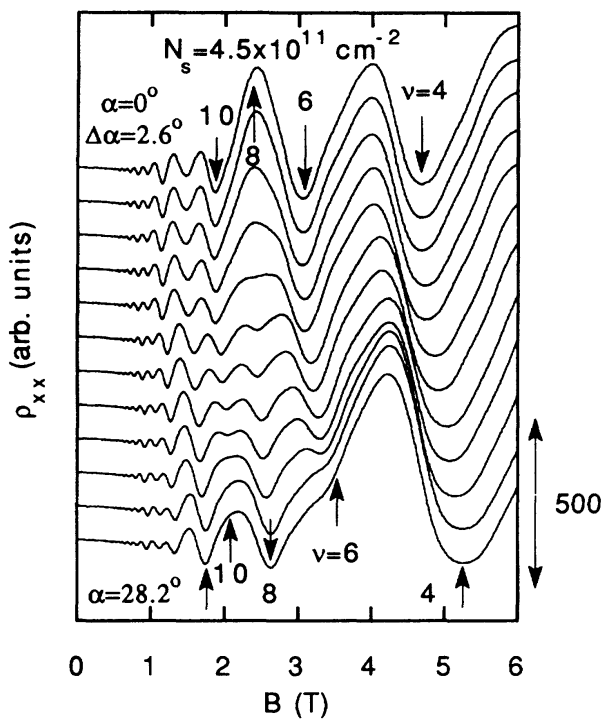

FIG. 1. Experimental magnetoresistance $\rho_{x x}$ for constant $N_{s}=4.5 \times 10^{11} \mathrm{~cm}^{-2}$ and various tilt angles $\alpha$. The curves are vertically offset with respect to each other for clarity. The arrows indicate the positions of the filling factors.

relative modest electron mobility $\left(\mu=100000 \mathrm{~cm}^{2} / \mathrm{V} \mathrm{s}\right)$.

Via a suitable gate bias the carrier density in Fig. 1 is chosen such that the quantum Hall state for $v=8$ is suppressed (see also Ref. 12) for $\alpha=0$. In this case the $|i, n\rangle=|0,2\rangle$ and $|i, n\rangle=|1,1\rangle$ states are degenerate at the Fermi energy. This situation is reflected in a maximum in the magnetoresistance $\rho_{x x}$ at $v=8$ and a missing quantum Hall plateau in the Hall resistance $\rho_{x y} .{ }^{12}$ Since the existence of a quantum Hall plateau in $\rho_{x y}$ is always correlated with a corresponding minimum in $\rho_{x x}$ we will restrict the discussion to the behavior of $\rho_{x x}$. For increasing tilt angles the maximum in $\rho_{x x}$ at $v=8$ gradually turns into a minimum and is well pronounced at $\alpha=28.2^{\circ}$. At the same time the neighboring minima at $v=6$ and $10 \mathrm{ex}-$ hibit the opposite behavior and disappear for large tilt angles. This fascinating observation is a direct manifestation of the level anticrossing in tilted magnetic fields. It allows the immediate determination of the single-particle subband separation from magnetotransport experiments. We can observe this effect each time a quantum Hall plateau is suppressed at $\alpha=0$ and is recovered for finite $\alpha$. However, the strength of the resonant subband-Landaulevel coupling depends on the particular subband structure and the values of the respective quantum numbers. A detailed analysis of this problem is possible on the basis of simple algebra with the quantum numbers. This is not the focus of the present paper and we will restrict the following discussion on the situation of filling factor 8 as depicted in Fig. 1.

To understand the problem in a more quantitative way we have solved self-consistently Schrödinger and Poisson's equation for $B=0$. Cyclotron resonance measurements on PQWs (Refs. 16 and 17) indicate that deviations of the cyclotron effective mass caused by the parabolic Al profile in the well are smaller than $1 \%$. It is therefore justified to neglect nonparabolicity effects due to the varying $\mathrm{Al}$ con- tent within the potential profile. For the calculation a constant effective mass of $m^{*}=0.067 m_{0}$ has been used. It has been shown that the subband populations as well as the subband energies $E_{i}$ depend on the magnetic field. ${ }^{18}$ The magnitude of these effects is comparable to the typical Landau-level width $(\sim 0.5 \mathrm{meV})$ and therefore influences the detailed suppression behavior of a quantum Hall plateau. Here the decisive quantity is the level repulsion due to the parallel component of the magnetic field. Since this level separation is larger than typical effects of the magnetic field on the self-consistent subband structure itself it is justified to use a harmonic approximation for the evaluation of the level anticrossing even though the self-consistent potential is no longer parabolic. Consequently the subband separation $E_{10}=E_{1}-E_{0}$ obtained from the self-consistent calculation at $B=0$ is used to model a parabolic potential with characteristic frequency $\hbar \omega=E_{10}$. This case can be solved analytically in a tilted magnetic field ${ }^{13,14}$ and the level structure is obtained. We only consider two electrical subbands in accordance with the experimental situation. The magnetoconductance $\sigma_{x x}$ is then calculated according to Ref. 11 with

$$
\sigma_{x x}=e^{2} / h \sum_{i, n}\left(n+\frac{1}{2}\right) \exp \left[-\left(E_{F}-E_{i, n}\right)^{2} / \Gamma^{2}\right] .
$$

The linewidth of the broadened Landau levels is chosen as $\Gamma=(0.5 \mathrm{meV}) \times(B(T))^{1 / 2}$, a value that described reasonably well magnetocapacitance experiments on samples with similar mobilities. ${ }^{19}$ Consequently, there are no adjustable parameters. Since the Hall resistance $\rho_{x y}$ behaves almost classically in the regime of interest due to the suppression of the Hall plateaus, it is possible to assume $\sigma_{x y}=-e N_{s} / B$ for the Hall conductivity, which then allows the magnetoresistance $\rho_{x x}$ to be calculated. The energies $E_{i, n}$ are calculated from the parabolic model ${ }^{13,14}$ and the position of the Fermi energy $E_{F}$ at a given magnetic field $B$ is evaluated using the $E_{i, n}$ and keeping $N_{s}$ constant. For $\alpha=0$, the energy spectrum is simply given by $E_{i, n}=E_{i}+\left(n+\frac{1}{2}\right) \hbar \omega_{c}$ with $\omega_{c}=e B / m$. This theoretical method has proven very useful in explaining experiments for $\alpha=0$ (e.g., Refs. 12 and 20).

For tilted magnetic fields $\alpha \neq 0$ hybrid electric-magnetic subbands are formed and $i$ and $n$ are no longer good quantum numbers, but new quantum numbers arise for a rotated coordinate system. ${ }^{13,14}$ Consequently, the carrier density of a given subband is not a well-defined quantity any more, especially in the vicinity of a resonant level anticrossing. Nevertheless, we will, in the following discussion, stick to $i$ and $n$ even for the tilted field case $\alpha \neq 0$, to accomplish a transparent picture of the experimental situation. Since $\sigma_{x x}$ and therefore $\rho_{x x}$ depend sensitively on $i$ and $n$, care must be taken with the application of the transport theory" to the tilted field case. Nevertheless, the position of the Fermi energy in the gap between the energy levels results in a minimum of $\rho_{x x}$, which is therefore well defined. More problematic are the heights and positions of the maxima in $\rho_{x x}$, since they depend more sensitively on the subband carrier densities and the quantum numbers. The results of this calculation are plotted in Fig. 2 in the same way as the corresponding experimental data in Fig. 1 is presented. The overall conformity 


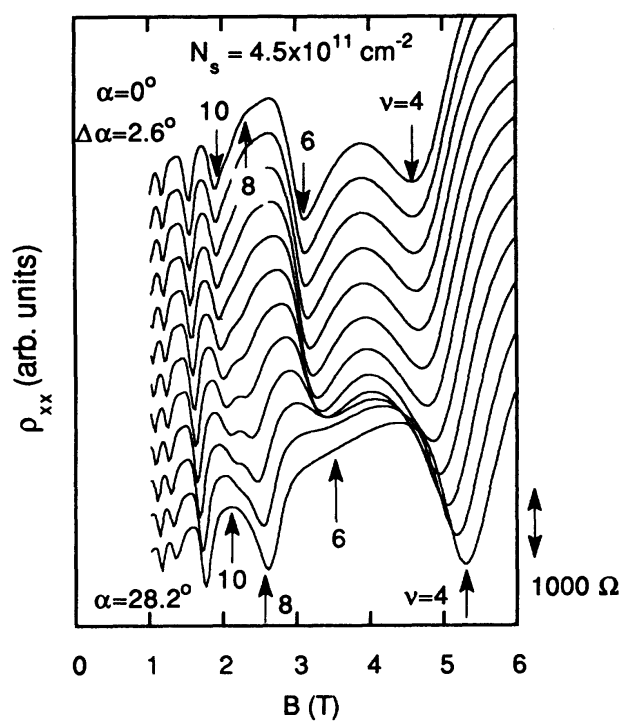

FIG. 2. Theoretical results presented in the same way as the experimental data in Fig. 1. The curves are plotted for only $B>1 \mathrm{~T}$ where a decent number of Landau levels has to be considered for the calculation.

is overwhelming reflecting the justified approximations made in the calculations. The suppressed minimum (for $\alpha=0)$ of $v=8$ is clearly recovered for large tilt angles while the neighboring minima at $v=6$ and 10 endure the opposite fate. The $\rho_{x x}$ scale is indicated on the right-hand side of Figs. 1 and 2 . In the calculation the amplitude of the magnetoresistance oscillations is in general larger due to the negligence of localized states in the description of the Landau levels. The $\rho_{x x}$ maximum between $v=4$ and 6 is clearly underestimated in the calculation because of the inappropriate use of the quantum numbers in the transport calculation. This does not, however, influence the position of the minima and the reappearance of $v=8$.

The calculated energy-level structure for three typical situations is presented in Fig. 3. For the untilted case $\alpha=0$ the linear Landau levels cross each other and, for $v=8$, the Landau level $n=1$ of the upper subband $i=1$ is degenerate with the Landau level $n=2$ of the lower subband $i=0$. For the intermediate situation, $\alpha=15.4^{\circ}$, these two Landau levels start to repel each other leading to a recovery of the minimum in $\rho_{x x}$ at $v=8$. The anticrossing occurs for $E_{10}=\hbar \omega_{c}$. From the experiment in Fig. 1 we deduce the magnetic field, where the minimum corresponding to filling factor $v=8$ reappears and conclude that $E_{10}=4.2 \mathrm{meV}$ in close agreement with the theoretical result $(4.4 \mathrm{meV})$ from the self-consistent calculation. The design energy of the bare harmonic-oscillator potential is $10.7 \mathrm{meV}$ which has been confirmed by optical intersubband spectroscopy. The observed intersubband separation of $4.2 \mathrm{meV}$ indicates the flattening of the potential with increasing electron number and the good theoretical understanding of the self-consistent process. Simultaneously, the levels $|i, n\rangle=|0,3\rangle$ and $|i, n\rangle=|1,1\rangle$ (corresponding to $v=10$ ) move closer together. The repulsion is reduced because there $\Delta n=2$. In first-order perturbation theory this level anticrossing is identically

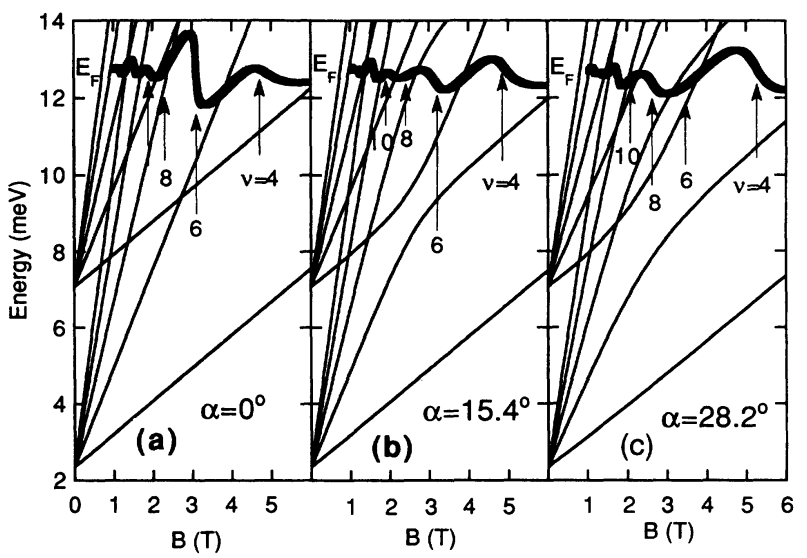

FIG. 3. Calculated energy-level spectra for three different tilt angles and $N_{s}=4.5 \times 10^{11} \mathrm{~cm}^{-2}$. The hybrid electric-magnetic subbands are plotted by thin lines, the behavior of the Fermi energy is indicated by the thick line. The lower (upper) set of Landau levels extrapolates for $B=0$ to $\left(E_{1}-E_{0}\right) / 2$ $\left[\left(E_{1}-E_{0}\right) 3 / 2\right]$, respectively.

zero. The same is true for the levels $|0,2\rangle$ and $|1,0\rangle$. Therefore the minimum corresponding to $v=6$ vanishes for large tilt angles, where the two levels are closer than the width of the Landau levels. Of course, in the limit of large tilt angles $\alpha>50^{\circ}$, all levels repel each other strongly and all filling factors are again recovered as has been observed in Ref. 21 . In the present calculation the diamagnetic shift of the electric subband energies caused by the parallel field component is inherently considered. It is, however, a weak effect $(<0.5 \mathrm{meV})$ and the level anticrossing is the dominant cause of the recovery of the $v=8$ minimum in $\rho_{x x}$.

A number of transport experiments in tilted magnetic fields have been reported in the literature, e.g., Refs. 21-26, many of them devoted to the study of the fractional quantum Hall effect, e.g., Refs. 22 and 23. The transport properties of a parabolic well were investigated in Ref. 21 for large tilt angles $\alpha>45^{\circ}$ and the magnetic depopulation of the subband was observed similar to Ref. 26, where the influence of parallel fields was studied. In Ref. 24 a 50-nm-wide bulk doped quantum well was investigated and a bulklike behavior of the energy levels was found, once the magnetic quantization energies dominate the electric ones. A usual $\mathrm{Al}_{x} \mathrm{Ga}_{1-x}$ As heterostructure containing a 2 DEG revealed activation energies in tilted magnetic fields that allowed estimations for the nonresonant coupling of the Landau levels in the system. ${ }^{25}$ The resonant subband-Landau-level coupling, as it is reported in this paper, has not, to our knowledge, been observed so far in transport experiments. A crucial ingredient is the very particular subband structure of a parabolic well that can neither be realized in a bulk doped quantum well ${ }^{24}$ nor in a usual 2 DEG. The important fact that the subband separations in a PQW decrease for increasing carrier density is decisive for a relatively large amount of carriers in the upper subband. Thus a higher subband is not depopulated in a magnetic field, as it usually occurs in 2DEGs, but can gain carriers at the cost of 
the lower subband.

In far-infrared transmission experiments the intersubband transitions are governed by the depolarization effect. ${ }^{4}$ To date mainly the coupling of the $|0,1\rangle$ and $|1,0\rangle$ levels has been investigated ${ }^{1,2,4}$ and, for large tilt angles, also the $|0,2\rangle$ to $|1,0\rangle$ coupling. ${ }^{3}$ In a PQW the different levels cannot be resolved since the resonance energies are dominated by the depolarization effect. ${ }^{5-7,10}$ In the present transport experiment, however, we can observe directly the anticrossing of the single-particle energies for a whole series of levels, e.g., $|0,2\rangle$ to $|1,1\rangle$ coupling for the case of suppressed $v=8$ at $\alpha=0,|0,1\rangle$ to $|1,0\rangle$ corresponding to $v=4$, and $|0,3\rangle$ to $|1,2\rangle$ coupling for $v=12$. This unique experimental situation allows the direct evaluation of the single-particle subband separations from a transport experiment.

In conclusion we have presented transport experiments in tilted magnetic fields that provide immediate access to the single-particle energy spectrum of a parabolic quantum well. In tilted magnetic fields the resonant coupling of occupied Landau levels belonging to different electric subbands results in the recovery of quantum Hall states that are suppressed for magnetic fields perpendicular to the electron system.

We thank J. P. Kotthaus and D. Wharam for stimulating discussions. This work was supported by the Air Force and by Esprit. We gratefully acknowledge this financial support.
'Z. Schlesinger, J. C. M. Hwang, and S. J. Allen, Jr., Phys. Rev. Lett. 50, 2098 (1983).

${ }^{2}$ G. L. J. A. Rikken, S. Sigg, C. J. G. M. Langerak, H. W. Myron, J. A. A. J. Perenboom, and G. Weimann, Phys. Rev. B 34, 5590 (1986).

${ }^{3}$ A. D. Wieck, J. C. Maan, U. Merkt, J. P. Kotthaus, K. Ploog, and G. Weimann, Phys. Rev. B 35, 4145 (1987).

${ }^{4}$ K. Ensslin, D. Heitmann, and K. Ploog, Phys. Rev. B 39, 10879 (1989).

${ }^{5}$ K. Karrai, H. D. Drew, H. W. Lee, and M. Shayegan, Phys. Rev. B 39, 10426 (1989).

${ }^{6}$ K. Karrai, X. Ying, H. D. Drew, and M. Shayegan, Phys. Rev. B 40, 12020 (1989).

${ }^{7}$ A. Wixforth, M. Sundaram, K. Ensslin, J. H. English, and A. C. Gossard, Surf. Sci. (to be published).

${ }^{8}$ W. P. Chen, Y. J. Chen, and E. Burstein, Surf. Sci. 58, 263 (1976)

${ }^{9}$ S. J. Allen, Jr., D. C. Tsui, and B. Vinter, Solid State Commun. 20, 425 (1976).

${ }^{10}$ L. Brey, N. F. Johnson, and B. I. Halperin, Phys. Rev. B 40, 10647 (1989).

"R. R. Gerhardts, Surf. Sci. 58, 227 (1976).

${ }^{12}$ K. Ensslin, M. Sundaram, A. Wixforth, J. H. English, and A. C. Gossard, Phys. Rev. B 43, 9988 (1991).

13J. C. Maan, in Two Dimensional Systems, Heterostructures, and Superlattices, edited by G. Bauer, F. Kuchar, and $\mathrm{H}$. Heinrich (Springer-Verlag, Berlin, 1984), p. 183.
${ }^{14}$ R. Merlin, Solid State Commun. 64, 99 (1987).

${ }^{15}$ M. Kroeker and U. Merkt, Solid State Commun. 69, 699 (1989).

${ }^{16}$ A. Wixforth, M. Sundaram, D. Donelly, A. C. Gossard, and M. Shayegan, Surf. Sci. 228, 9732 (1990).

${ }^{17}$ K. Karrai, M. Stopa, X. Ying, H. D. Drew, S. Das Sarma, and M. Shayegan, Phys. Rev. B 42, 9732 (1990).

${ }^{18}$ K. Ensslin, M. Sundaram, A. Wixforth, and A. C. Gossard, Surf. Sci. (to be published).

${ }^{19}$ See, for example, K. Ensslin, D. Heitmann, R. R. Gerhardts, and K. Ploog, Phys. Rev. B 39, 12993 (1989).

${ }^{20}$ G. Gobsch, D. Schulze, and G. Paasch, Phys. Rev. B 38, 10943 (1988).

${ }^{21}$ M. Shayegan, T. Sajoto, J. Jo, M. Santos, and H. D. Drew, Phys. Rev. B 40, 34476 (1989).

${ }^{22}$ R. J. Haug, K. v. Klitzing, R. J. Nicholas, J. C. Maan, and G. Weimann, Phys. Rev. B 36, 4528 (1987).

23J. P. Eisenstein, R. Willett, H. L. Störmer, D. C. Tsui, A. C. Gossard, and J. H. English, Phys. Rev. Lett. 61, 997 (1988).

${ }^{24}$ J. Yoshino, H. Sakaki, and T. Hotta, Surf. Sci. 142, 326 (1984).

25 J. J. Koning, R. J. Haug, H. Sigg, K. v. Klitzing, and G. Weimann, Phys. Rev. B 42, 2951 (1990).

${ }^{26}$ E. G. Gwinn, R. M. Westervelt, P. F. Hopkins, A. J. Rimberg, M. Sundaram, and A. C. Gossard, Phys. Rev. B 39, 6260 (1990). 